\title{
Data Collection and Analysis Software Development for Rotor Dynamics Testing in Spin Laboratory
}

\author{
Ali Abdul-Aziz ${ }^{1}$, Daniel Arble ${ }^{2}$ and Mark Woike ${ }^{3}$ \\ ${ }^{1}$ Kent State University, Kent, Ohio, 44242 \\ ${ }^{2}$ University of Maryland, College Park, MD, 20740 \\ ${ }^{3}$ NASA Glenn Research Center, Cleveland, Ohio, 44135
}

\begin{abstract}
Gas turbine engine components undergo high rotational loading another complex environmental conditions. Such operating environment leads these components to experience damages and cracks that can cause catastrophic failure during flights. There are traditional crack detections and health monitoring methodologies currently being used which rely on periodic routine maintenances, nondestructive inspections that often times involve engine and components dis-assemblies. These methods do not also offer adequate information about the faults, especially, if these faults at subsurface or not clearly evident. At NASA Glenn research center, the rotor dynamics laboratory is presently involved in developing newer techniques that are highly dependent on sensor technology to enable health monitoring and prediction of damage and cracks in rotor disks. These approaches are noninvasive and relatively economical. Spin tests are performed using a subscale test article mimicking turbine rotor disk undergoing rotational load. Non-contact instruments such as capacitive and microwave sensors are used to measure the blade tip gap displacement and blade vibrations characteristics in an attempt develop a physics based model to assess/predict the faults in the rotor disk. Data collection is a major component in this experimental-analytical procedure and as a result, an upgrade to an older version of the data acquisition software which is based on LabVIEW program has been implemented to support efficiently running tests and analyze the results. Outcomes obtained from the tests data and related experimental and analytical rotor dynamics modeling including key features of the updated software are presented and discussed.
\end{abstract}

Keywords: Spin Testing, LabVIEW, Crack Detection, Rotor, Jeffcott, Sensors, Health Monitoring

\section{INTRODUCTION}

Efforts by engine companies and government agencies such as NASA are continuously underway to make aircraft engines safer, efficient and reliable. A partnership between the NASA Aviation Safety program (AVSP), the Federal Aviation Administration, Department of Defense, and aviation industry to develop techniques to monitor the health of aircraft turbine engine is ongoing to develop techniques that support maintaining the health of these engines and in particular their hot section components ${ }^{1}$. Monitoring the performance of these components like the blades, disks and rotors allow a close up inspection which helps in reducing associated cost and engine down time. This further contributes to improving the performance of the engine and in particular the efficiency. Such improvement has been demonstrated by reducing the blade tip clearance, the smaller the tip clearance, the more efficient is the engine ${ }^{1-2}$. Additionally, other factors such as foreign objects and other operating conditions could lead to fatigue and cracking in the rotor disk. If these damages are not contained and controlled, fragmentation from the damaged components can lead to complete engine failure and catastrophic results. Therefore, the need for advanced inspection technology to pre-detect such pre-existing anomalies is highly essential to maintaining a safer engine and more reliable aircrafts.

Currently, monitoring and inspection techniques are limited to standard nondestructive evaluation applications that focuses on evaluating material's discontinuities and other defects that are matured and are prominent to failure. Work is containing here at NASA Glenn to integrate other means of inspection using non-contact sensor technology such as microwave and capacitive type approach. This is being implemented via supportive combined experimental and analytical activities by conducting highly specialized spin tests on a rotor disk turbine engine like test article followed by modeling validation. The testing is performed over a precision rig system to monitor the rotor performance under rotational speed loading up to $12,000 \mathrm{Rpm}$ thru observing the blade tip displacement variation and detecting a pre-determined damages such as an artificially induced notch or a crack ${ }^{3}$. 
This paper focuses on presenting implemented and added updates into the LabVIEW virtual instrument (VI) data acquisition module used to measure data recording and analyses. In addition, related results obtained from the spin testing and the rotor dynamics model are also included. An improved user interface and supplementary capabilities to display main pieces of information, such as the net rotor blade displacement, vibration response, eddy current data and other values are discussed and presented.

\section{ROTOR DYNAMIC LABORATORY DESCRIPTION}

The rotor dynamics spin laboratory at NASA Glenn is designed such as it has the capabilities to spin a subscale test article rotor disk with a maximum outside diameter of $23.495 \mathrm{~cm}(9.25 \mathrm{in})$ at a rotational speed up to 15,000 Rpm. It is equipped with high power custom made electric motor that can rotate the disk to the desired speed. Figure 1 shows the full configuration of the current spin rig system. The current test rotor disks are comprised of nickel-base alloy Haynes X-750 and weigh 10.75 lbs., with 32 equidistant blades about the disk circumference ${ }^{3}$. A pair of capacitive probes is used to measure the blade tip clearance during a test, acquiring data at a scan rate of $1 \mathrm{MHz}$ and a pair of eddy current probes scan at a rate of $204.08 \mathrm{kHz}$ are used to obtain data to compute the shaft centerline displacement. A HT-5200 digital tachometer records the current rpm of the test and is used to sync the capacitive and eddy current probe data. Controlling the test process is a managed by a control panel that allow set up of the rpm and acceleration input of the spinning disk from an adjacent room, where motor temperature is also monitored.

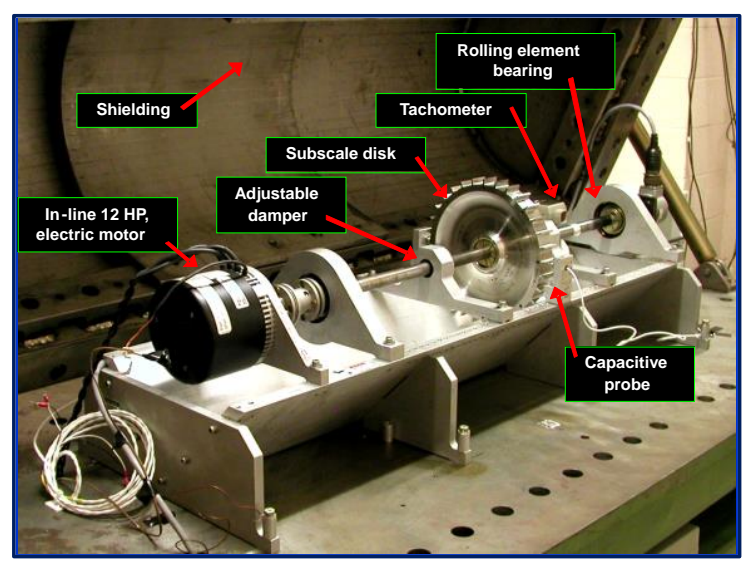

a) High precision spin laboratory

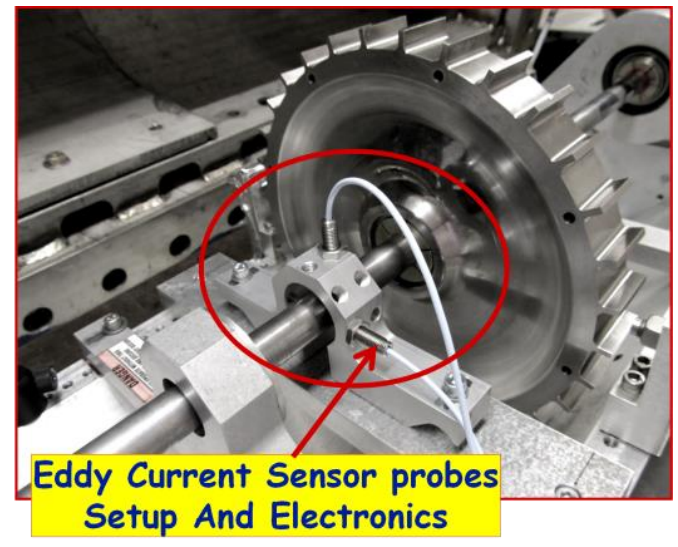

b) Eddy Current sensor-disk rotor set up

Figure 1. Photo of the NASA Glenn Spin Rig set up showing various key components.
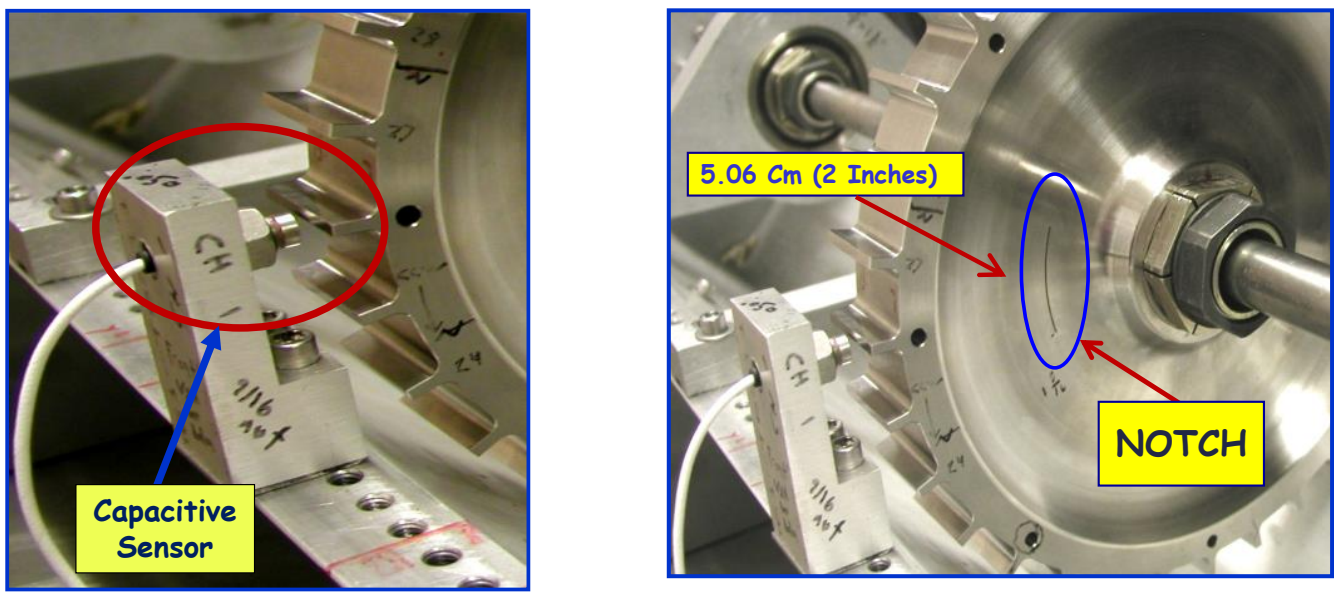

Figure 2. Photo of the High-performance capacitive displacement sensor and the Notched Rotor Disk 


\section{Data Acquisition System Description}

The data acquisition system used in the spin experiments is dependent both the actual national instrument hardware and software supported by LabView code ${ }^{4-5}$. A custom made module was developed and designed to help in acquiring needed data for analysis management and test calibration. The module constitutes of a Virtual Instrument (VI) Graphical User Interface (GUI) based software panel, see Figure 3. This VI is essential to the data acquisition and analysis routine. It has the capability to allow for the automation of data logging, processing, reduction, and display of results. These four processes are accomplished in the five main modules highlighted in Figure 3.

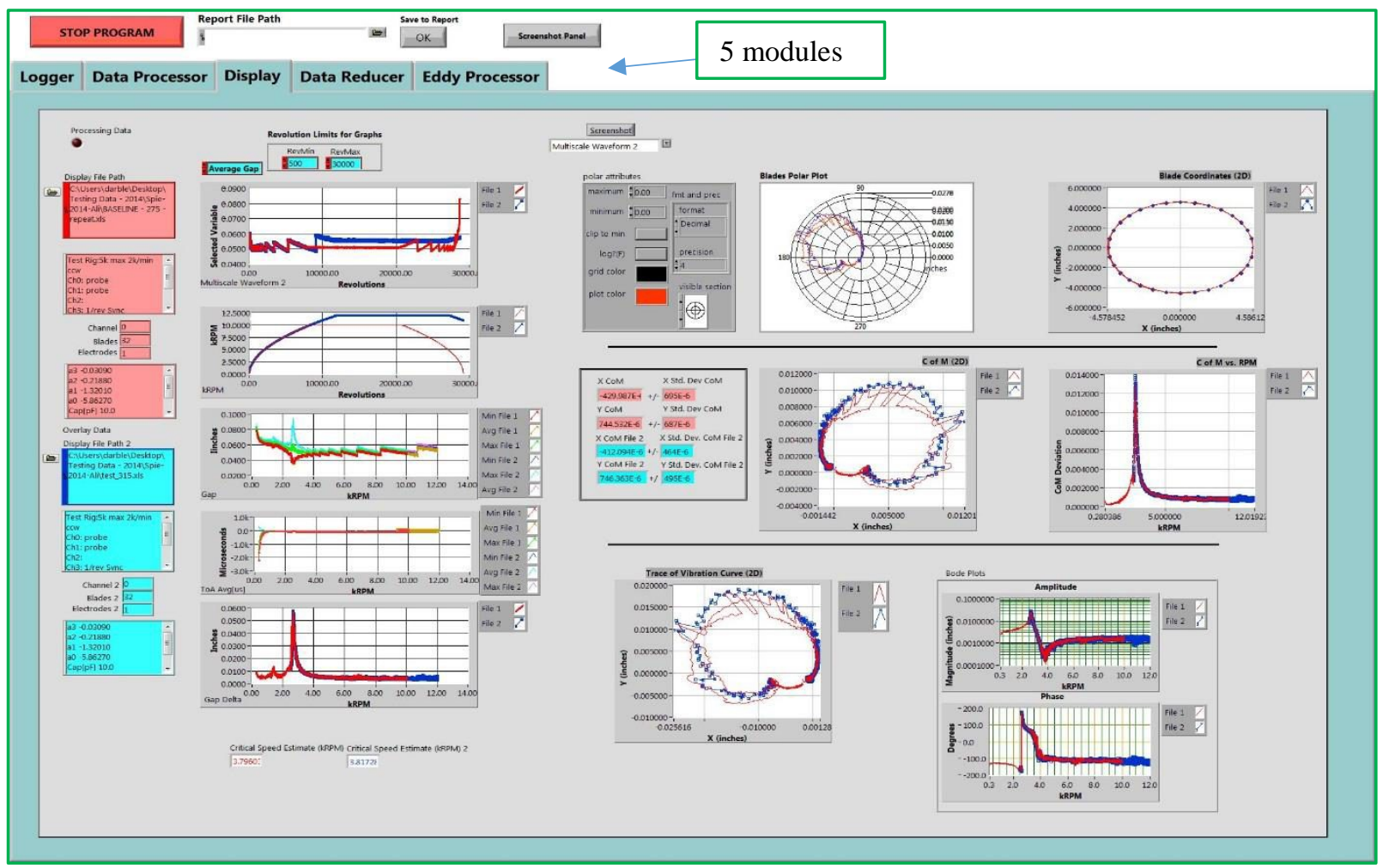

Figure 3. Updated Virtual Instrument LabView data acquisition module.

The Logger module records the data as a technical data management streaming (TDMS) file format ${ }^{4}$. Following the completion of data logging, the Data Processor module reads speed data from the tachometer, eddy current sensors ${ }^{6}$, and the raw voltage data from the capacitive probes. Blade tip clearance, vibrational magnitude, center of mass, and other information are recorded and calculated. The output is presented in excel spreadsheets for use in the Display and Data Reducer modules. The Eddy Processor module reads in the eddy current data to obtain the changes in the shaft centerline position which is used in calculating the net blade tip displacement.

\section{Data Acquisition Module Implemented changes and updates}

The data acquisition module was subjected to major changes, improvements to meet the test requirements and the complexity of the experimental setup. A plan summarizing these updates and changes was created and subsequently implemented. The original version of the software was limited, it was not as user friendly and was confined to minimum set of functionalities and capabilities. For instance, it did not provide any indication of the Logger progress during testing, the Data Reducer was not capable of operating on the eddy current sensor excel data files, and image capture was not available.

Meanwhile, the updated version was modified to account for a more robust eddy current data processor, an ability to capture images and screen shots in real time or while the VI is operating and running. In the prior version, capturing a screen shot required the use of a third-party software. Now, the user may choose to capture an image of the entire front panel or an individual graph, display, or chart anytime during the testing process from within the 
LabVIEW-VI module display. The updates allowed the user to monitor the test progress thru an indicator from the logger module until completion. In addition, the user is now able to view the timing display during the test. This permitted coordinating the operation of the VI with the control panel to more efficiently obtain the desired rpm profile. Further, a more customized option was created for the raw eddy current data. It can now be recorded to an excel spreadsheet in the Data Processor module, allowing the user to access the data without the need to use the VI. Additional feature was also employed which allowed the eddy current data to be reduced by averaging a specified selected set of data points without jeopardizing the accuracy of these data due to the high sampling rate. This permitted the reduced eddy data to be utilized in the Eddy Processor at a selected rpm value or blade number. This averaging process can also be used on the blade vibrations response as well.

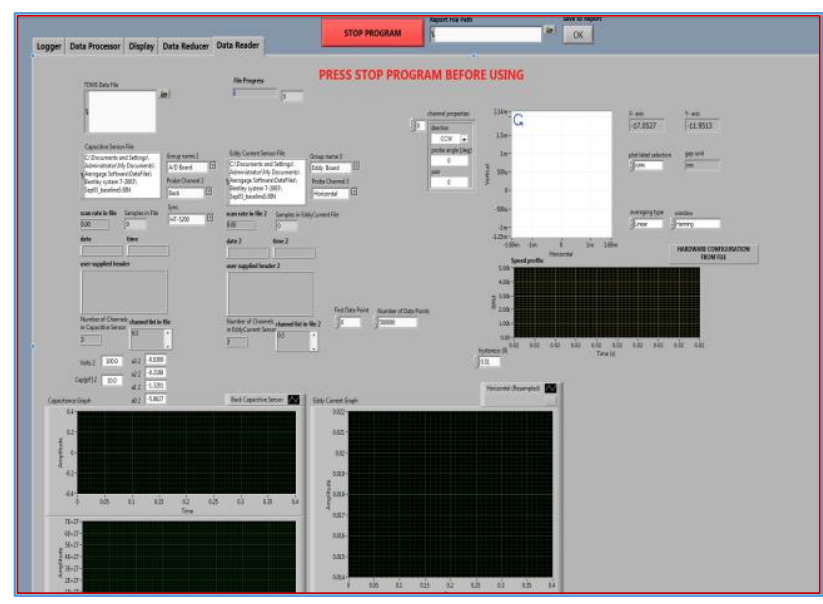

a) Old module

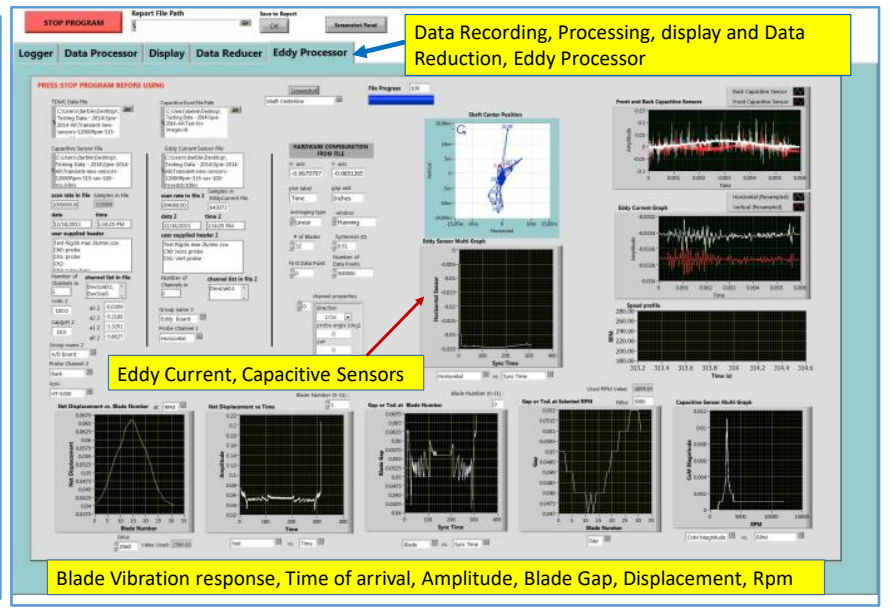

b) Updated module

Figure 4. Original and Updated screen shots of the Eddy Current Module.

The net displacement of the disk is calculated and displayed, taking into account the positional shift in the shaft centerline. Center of mass, gap, and vibration vector information is automatically plotted as well. The additions of these graphs can be seen in Fig. 4 (b). Automatic removal of faulty data from sensors that occurs at startup was implemented, providing a clearer representation of data in the displays of the Eddy Processor module. The VI was modified to be compatible with the newest version of LabVIEW available, LabVIEW 2014. The previous version was based on the LabVIEW 2009 version. Drivers for the National Instruments' data acquisition hardware were equally updated as well. This allowed for the VI and devices to be supported on any new LabVIEW software versions over the next four years ${ }^{5}$, which may be necessary if a new file format that improves the currently used TDMS format is released.

\section{Rotor dynamics model updates}

Analytical modeling is essential in validating the experimental findings, as a result, a parallel analytical rotor dynamics modeling is performed to check features and parameters that may influence the performance of the rotor/experimental rig and also to help better development of the crack detection methodology. The theory is based on a Jeffcott rotor concept ${ }^{7}$. The model is simplified such as it acknowledges the presence of an unbalanced rotor disk with massless elastic shaft in radially rigid bearings. It further assumes that the system is symmetric and the whirling speed and rotational speed will be the same and we have a synchronous whirl. A basic mathematical description of the model is shown in the following equations ${ }^{7-10}$;

$$
\begin{aligned}
& I=\frac{\pi R^{4}}{4} \\
& \omega_{n}=\frac{60}{2 \pi} \sqrt{\mathrm{k} / \mathrm{m}} \\
& \omega_{\text {crit }}=\frac{60}{2 \pi} \sqrt{\mathrm{k} / \mathrm{m}\left(1-2 \zeta^{2}\right)}
\end{aligned}
$$




$$
k=\frac{86 E I}{L^{3}}
$$

$$
\stackrel{e}{R} \text { whirl } 2_{i} c_{1} \omega^{2}+\frac{+c_{2} \omega}{\sqrt{\left(1-r^{2}\right)^{2}+(2 \zeta r)^{2}}}
$$

$\theta=a \tan \left(\frac{2 \zeta r}{\left(1-r^{2}\right)}\right)$

$$
\alpha=a \tan \left(\frac{e_{c r} \sin (\beta)}{e_{0}+e_{c r} \cos (\beta)}\right)
$$
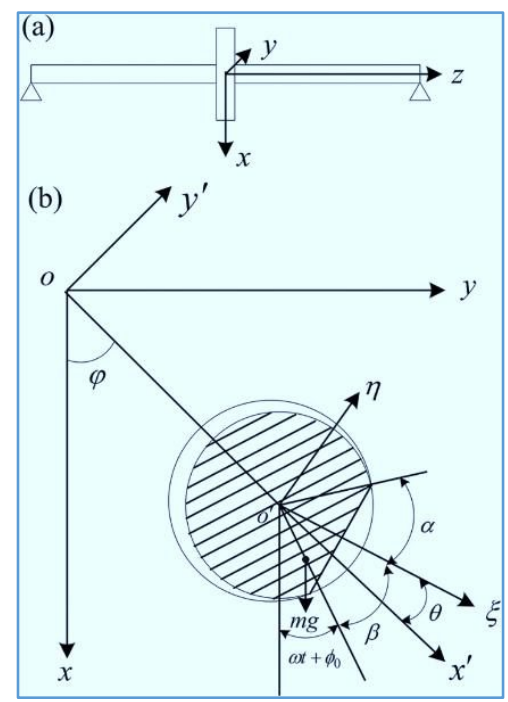

Figure 5. a) Jeffcott rotor model and b) back view of rotor mass diagram.

A LabVIEW VI module that runs the rotor model calculations and graphically displays the results was developed to accommodate the changes made on the data acquisition software. The coded model allows the variations of major variables such as damping ratio, mass, and eccentricity. The results can be further analyzed to permit observations of data outcome between cracked and undamaged disks.

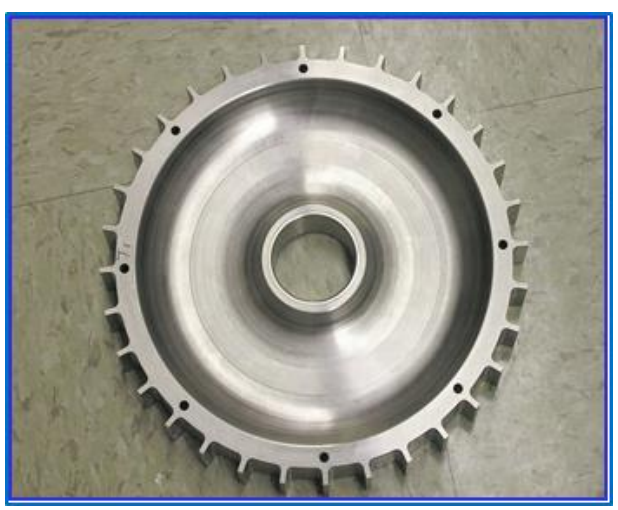

Fig 4. Undamaged Rotor Disk Testing Samples

A baseline model of the test rotors that was used to make comparisons against is characterized by a mass of $4.876 \mathrm{~kg}, \zeta=$ 0.23 , and $e_{0}=0.001$. With these parameters the baseline case resulted in a natural frequency of $2452 \mathrm{rpm}$ and critical frequency of $2593 \mathrm{rpm}$. Figures 5-7 shows the effects of varying rotor disk mass, damping ratio, and $e_{0}$ while keeping all other variables unchanged. 


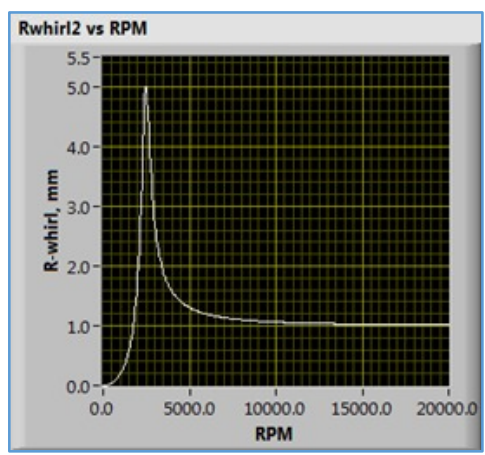

a. $\zeta=0.1$

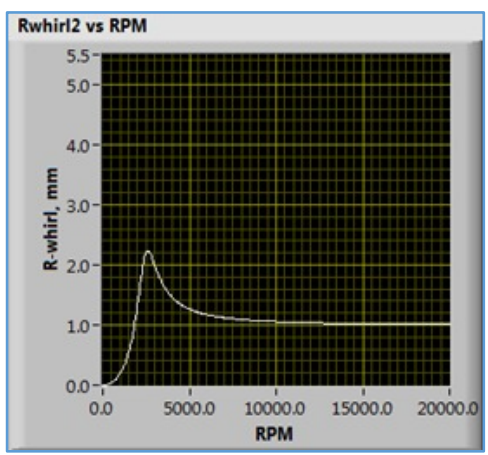

b. $\zeta=0.23$

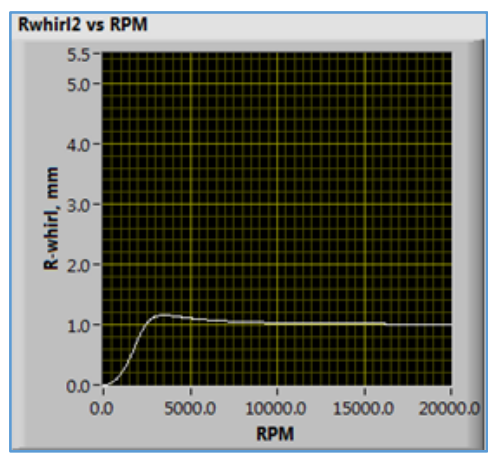

c. $\zeta=0.5$

Figure 5. Effects of Damping Ratio Variation on undamaged Rotor Whirl.

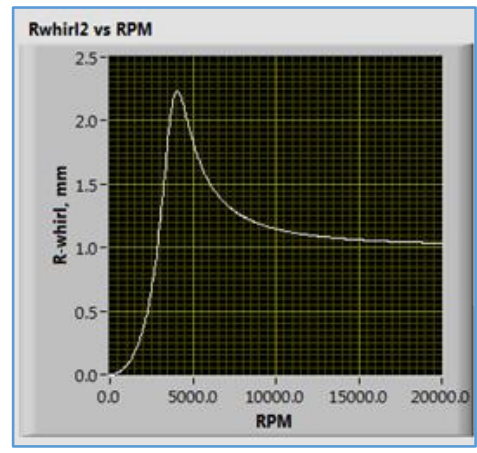

a. $\mathrm{m}=2.0 \mathrm{~kg}$

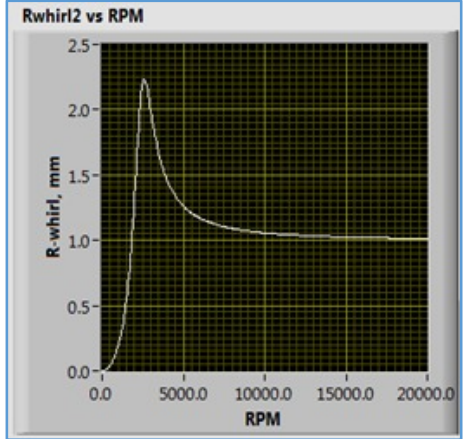

b. $\mathrm{m}=4.876 \mathrm{~kg}$

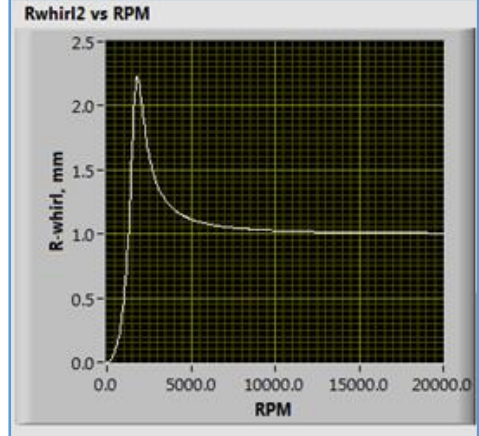

c. $\mathrm{m}=10.0 \mathrm{~kg}$

Figure 6. Effects of Rotor Mass Variation on Rotor Whirl

In Figure 5 maximum amplitude of $2.25 \mathrm{~mm}$ in the baseline case is increased to $5.0 \mathrm{~mm}$ when the damping ratio is decreased. The lower damping ratio caused the critical frequency to be reduced to $2477 \mathrm{rpm}$ from $2593 \mathrm{Rpm}$. Further in Figure 5, the increased damping ratio caused a decrease in the rotor whirl amplitude, to a maximum 1.15 from $2.25 \mathrm{~mm}$, and an increase in critical frequency to $3468 \mathrm{rpm}$ from $2593 \mathrm{rpm}$. Changes in the rotor disk mass caused a shift in natural and critical frequencies, indicated by the rpm value at which the graphs reach their maximum whirl amplitude, see Figure 6 . When the rotor disk mass was lowered to $2.0 \mathrm{~kg}$, the natural frequency and critical frequency rose to 3829 and $4049 \mathrm{rpm}$ respectively. Increasing the rotor disk mass to $10.0 \mathrm{~kg}$ lowers the natural and critical frequencies to 1712 and $1811 \mathrm{rpm}$. Higher rotor disk mass has led to a lower critical and natural frequencies.

The white plot represents the data of the phase for the undamaged disk, and the light and dark blue plots represent the phase data of a cracked disk at beta values of $90^{\circ}$ and $180^{\circ}$ respectively, See Figure 7 . The increase in initial eccentricity from 0.001 to 0.01 lowered the rate at which the phase increases until the rotor's natural frequency, was met and also lowered the maximum phase by $15^{\circ}$. After the rotor's natural frequency was met, the effects of initial eccentricity on phase become negligible. Reducing the initial eccentricity to 0.0 results in no change to phase as expected. This data allows for a better prediction of the experimental results from the spin rig tests in identifying the behavior of future rotors. 


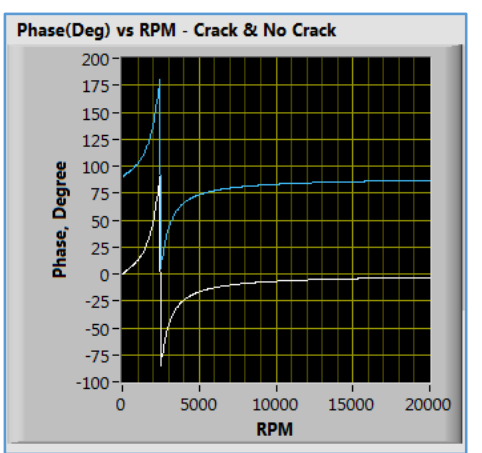

a) $\mathrm{e}_{\mathrm{o}}=0.0$

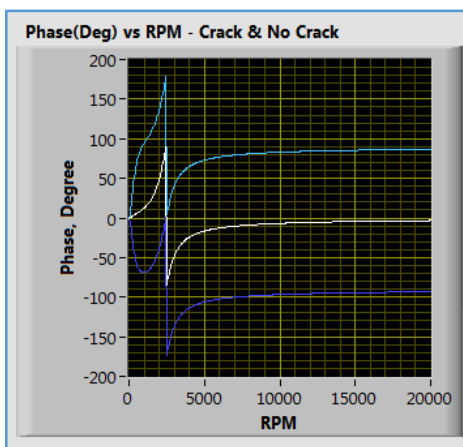

b) $\mathrm{e}_{\mathrm{o}}=0.001$

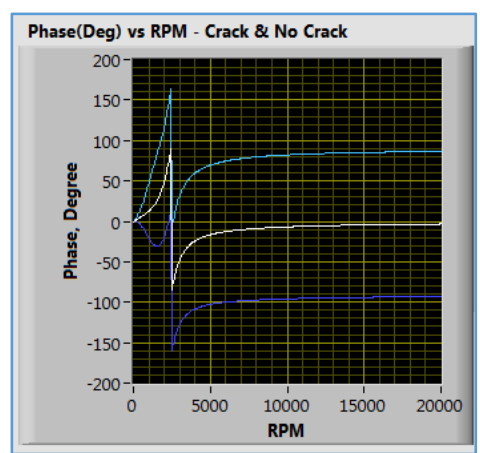

c) $\mathrm{e}_{\mathrm{o}}=0.01$

Figure 7. Effects of Initial Eccentricity Variation on Phase

\section{Spin test data}

Experimental tests were conducted by simulating the mission profile shown in Figure 8, reference 11 . The data produced from the experiments were generated for both rotors, the baseline with no notch and the damaged one with a notch, (Fig. 2). The updated LabView data acquisition software was used for the data collection and analyses. The data collected included the blade tip clearance, the vibration response and the shaft displacement. Representation of these responses are generated in terms of Bode plot format. Bode plots represent the gain and phase of a system as a function of frequency, or in our case as a function of rotational speed. This is referred to as the frequency domain behavior of a system.

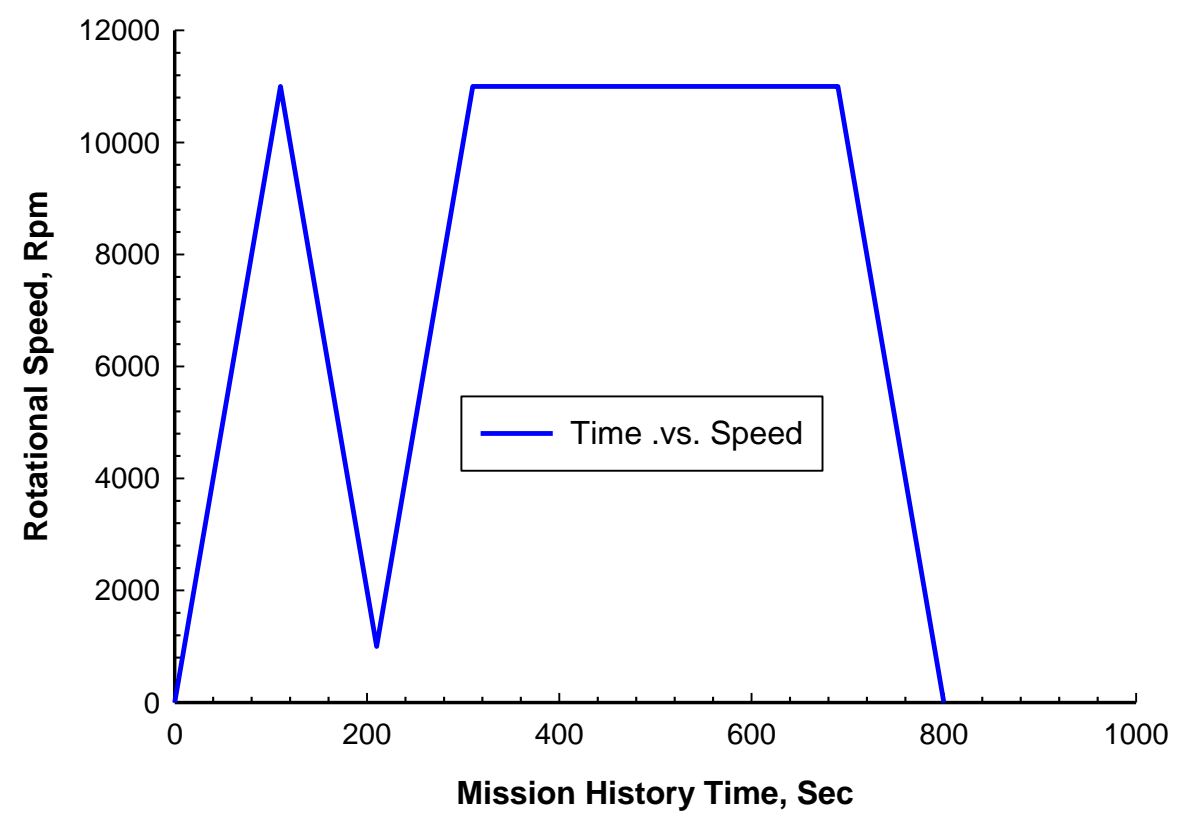

Figure 8. Engine mission history start-up- spike ignition profile ${ }^{9}$. 


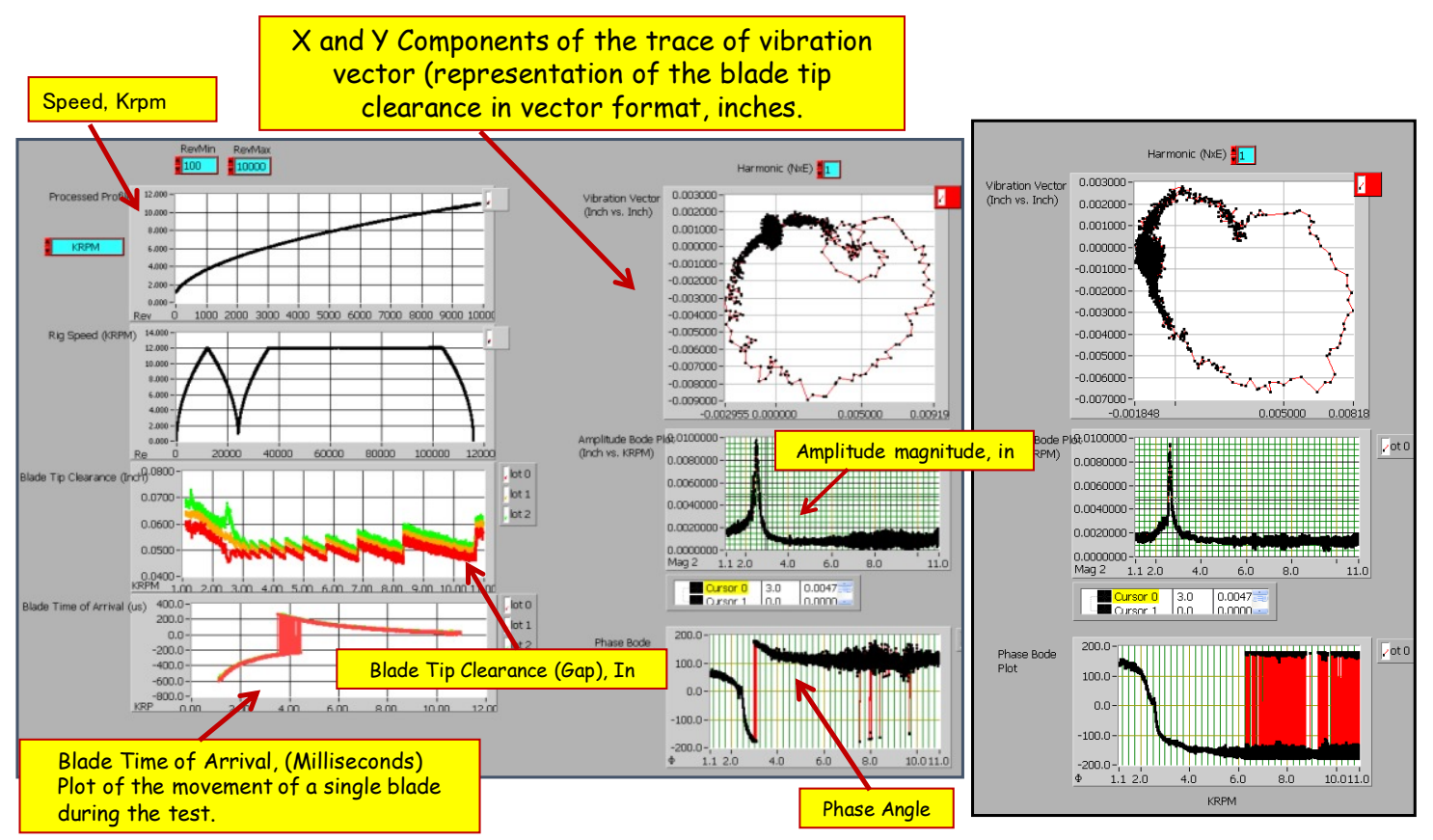

a) Rotor disk with notch

Figure 9. Vibration response due to rotational load.

Test data obtained are illustrated in Figures 9(a) and 9(b). They show the mission profile along with the trace vibration vector and the phase and amplitude response including notations used and their corresponding units. The plot further represents the trace of vibration response data for both the notched and un-notched rotor. A circular pattern type distribution is noted. This shows that the vibration response for the two structures responded rather differently for each rotor suggesting the existence of some type of variance between the two structures.

Ultimately, the goal is to run more tests so an amplitude response exhibiting an angular velocity $\left(\omega^{2}\right)$ rise settling past critical frequency is observed. Since such observation has indicated that a crack in the rotor would be present per reference ${ }^{12-21}$. Including more test data to verify such theory is beyond the scope of this paper, the purpose here is to present an overview of the rotor dynamics system capabilities at NASA Glenn and the updates made to its supportive data acquisition software. Furthermore, it is to show from these findings that the sensor system is able to assist in providing data needed to evaluate the health and performance of the rotor disk. However, additional investigation is warranted to endorse that such a structural health monitoring option is viable and dependable.

\section{CONCLUSIONS AND FUTURE WORK}

This paper presents a summary of updates made on a data acquisition software system used in the rotor dynamics laboratory at NASA Glenn Research center and it also offers supportive test results including analytical operation of a rotor dynamics model based on Jeffcott rotor approach. The following findings were perceived.

- The Virtual Instrument module (VI) user interface was updated and improved to meet the experimental rig and the testing requirements allowing for a more streamlined testing process. It was also modified to operate in the LabVIEW 2014 version.

- Adding Features such as indicating testing progress resulted in limiting the need to monitor testing time and it helped in providing a more user friendly experience with easier usability. An ability to record the eddy current sensor 
data and write that data to an excel file which can now be reduced to a more manageable size for graphing and analysis purposes was implemented.

- The screen capture utility was employed for both individual displays and the entire VI front panel. Moreover, updates to the rotor model were made which resulted in providing better understanding of the theoretical response of the rotor during testing, as well as the effect each variable has on the rotor vibration response.

- A key feature that remains to be implemented which is a mechanism to coordinate between the VI and the control box to accomplish the automation of the testing sequence and follow a customized user inputted speed profile. The later including reduction of computing resources in terms of processing time and memory which will aid in accelerating the testing process and analysis of the results are planned to be addressed in the future.

- The increase in initial eccentricity from 0.001 to 0.01 lowered the rate at which the phase increases until the rotor's natural frequency, was met and also lowered the maximum phase by $15^{\circ}$. After the rotor's natural frequency was met, the effects of initial eccentricity on phase become negligible. Reducing the initial eccentricity to 0.0 results in no change to phase as expected. This data allows for a better prediction of the experimental results from the spin rig tests in identifying the behavior of future rotors.

- Lastly, the combined sensors technology which included the capacitive and eddy current has supplemented the experimental data with ample information and allowed exploring the changes in the disk vibration response rather methodically.

\section{REFERENCES}

[1] Abdul-Aziz, A., et al., "Development of a Flaw Detection/Health Monitoring Scheme for Turbine Engine Rotating Components," Presented at the AIAA Infotech@Aerospace 2010, Atlanta, GA, 2010

[2] U.S. Department of Transportation, Federal Aviation Administration, "Engine Damage-Related Propulsion System Malfunctions," DOT/FAA/AR-08/24, 2008.

[3] Ali Abdul-Aziz, Mark R. Woike, John D. Lekki and George Y. Baaklini;" Development of a Flaw Detection/Health Monitoring Scheme for Turbine Engine Rotating Components"; Proceedings Smart Structures and Materials \& Nondestructive Evaluation and Health Monitoring; SPIE-1-12, March 11-15, San Diego, California. 2012.

[4] Abdul-Aziz, A., Curatolo, B.S., and Woike, M.R, "Improving Data Collection and Analysis Interface for the Data Acquisition Software of the Spin Laboratory at the NASA Glenn Research Center," NASA TM-217214, 2011.

[5] LabVIEW Software Package, Ver. 2014, National Instruments, Austin, TX, 2014

[6] http://www.ge-mcs.com/en/bently-nevada-sensors-and-transducers/proximity-probes/3300-xl-series.html.

[7] Rao, J., Rotor Dynamics, $2^{\text {nd }}$ ed., Wiley Eastern Limited, New Delhi, India, 1994

[8] S.Y.Youn et al.; "Introduction to Rotor Dynamics", DOI 10.1007/978-1-4471-4240-9_2, Springer-Verlag, London 2013.

[9] Jeffcott, H.H. "The lateral vibration of loaded shafts in the neighborhood of a whirling speed-The effect of want of balance", Phil. Mag., Series 6, Vol. 37, 19191, p. 304.

[10]Vance, J.M., "Rotordynamics of Turbomachinery," A Wiley-Inter-Science Publication, New York, 1988.

[11]DOT/FAA-AIAR-04/28;" Turbine Engine Fan Disk Crack Detection Test", September 2004, U.S. Department of Transportation, Federal Aviation Administration, Office of Aviation Research Washington, D.C. 20591.

[12]Wayne C. Hass and Michael j. Drumm;" Detection, Discrimination and Real-Time Tracking of Cracks in Rotating Disks", IEEE, 2002.

[13]Haase, W.C. and Drumm, M.J., "Detection, Discrimination, and Real-Time Tracking of Cracks in Rotating Disks," SPIE's NDE and Health Monitoring of Aerospace Materials and Civil Infrastructures, San Diego, California, March, 2002.

[14]Sekar, A.S., Prabhu, B.S., Condition Monitoring of Cracked Rotors Through Transient Response, Mechanism and Machine Theory, Vol. 33, Issue 8 (1998), p. 1167-1175.

[15]Bentlty, D.E, Detecting Cracked Shafts at Earlier Levels, Orbit Magazine, Bently Nevada, Vol. 3, No. 2, (1982). 
[16]Wauer, J., On the Dynamics of Cracked Rotors: A literature Survey, Applied Mechanics Review, Vol. 43(1), 1990. PP.1317.

[17]Sonnichsen, H.E. and Milatovic, B., “Detecting Anomalies in Rotating Components," United States Patent 6456945 B1, September 24, 2002.

[18]Lewicki, D.G, Emmerling, W.C., Altobelli, D., Seng, S., Frankenberger, C., and Fila, L., "TF41Engine Fan Disk Seeded Fault Crack Propagation Test," NASA/TM-2004-213092.

[19]Luo, H., Rodriguez, H., Hallman, D., and Corbly, D., “Disk Crack Detection for Seeded Fault Engine Test," NASA/CR 2004-213069.

[20]Ashok K. Koul and Raymond V. Dainty, "Fatigue Fracture of Aircraft Engine Compressor Disks", Rotating Equipment, Handbook of Case Histories in Failure Analysis, pp. 241-250, Volume 1, 2002.

[21]Sonnichsen, H. E., "Real-time Detection of Developing Cracks in Jet Engine Rotors”, www.testdevices.com, 0-78035846-5/00, IEEE, 2000.

$e_{0}=$ initial eccentricity, $\mathrm{mm}$

\section{Nomenclature}

$e_{c r}=$ notched induced eccentricity change, $\mathrm{mm}$

$\mathrm{m}=$ mass, $\mathrm{kg}$

$\mathrm{a}=$ distance between the center of the disk and its center of gravity, $\mathrm{mm}$

$\mathrm{R}=$ shaft radius, $\mathrm{cm}$

$\mathrm{E}=$ modulus of elasticity, $\mathrm{N} / \mathrm{m}^{2}$

$\mathrm{L}=$ shaft length, $\mathrm{m}$

$\mathrm{k}=$ bending stiffness of shaft, $\mathrm{N} / \mathrm{m}$

I $=$ moment of inertia

$\omega=$ Angular velocity, RPM

$\omega_{n} \quad=\quad$ Natural Frequency, $\mathrm{Hz}$

$\omega_{\text {crit }}=$ Critical Frequency, $\mathrm{Hz}$

$\zeta=$ damping ratio

b $=$ notch angle rel. to e e vector, rad

$\theta=$ phase angle, rad

$\alpha=$ phase angle contribution from, rad 